\title{
Wire antenna model of the vertical grounding electrode
}

\author{
D. Poljak \& S. Sesnic \\ University of Split, FESB, Split, Croatia
}

\begin{abstract}
A straight wire antenna model of the vertical grounding electrode has been presented in the paper. The formulation is based on the homogeneous integral equation of Pocklington type for half-space problems. The influence of a finitely conducting half-space is taken into account via the corresponding reflection coefficients. The Pocklington equation is solved both numerically and analytically. The numerical solution is carried out via the Galerkin-Bubnov scheme of the Indirect Boundary Element Method (GB-IBEM). Some illustrative computational examples are presented at the end.
\end{abstract}

Keywords: vertical grounding electrode, antenna model, Pocklington integral equation, boundary element method, analytical solution.

\section{Introduction}

Vertical grounding electrode is considered to be one of the key components of practical grounding systems, particularly for the protection of wind turbines [1] as they are extremely vulnerable to lightning strikes due to their special shape and isolated locations, mainly in high altitude areas. A typical realistic grounding system for wind turbines is rather complex and usually composed from rings, horizontal and vertical electrodes, respectively [1]. A frequency domain numerical/analytical antenna theory model of horizontal electrodes and ring electrodes as individual elements, has been carried out in [2] (Galerkin-Bubnov scheme of the Indirect Boundary Element Method (GB-IBEM) + analytical solution) and [3] (GB-IBEM), respectively. Vertical electrode, on the other hand, has not been analyzed as an individual element, to a greater extent, using antenna model and related numerical and analytical solution procedures. 
Though an electromagnetic transient analysis of complex grounding systems for wind turbines has been already reported elsewhere, e.g. in [1] where a combination of the ring, vertical rods and horizontal electrodes is considered, a detailed study of vertical electrode behavior, as an individual element, still remains rather important. Thus, of particular importance is the analytical analysis, as the complex practical configurations consisting of straight electrodes and ring incorporated in concrete blocks are rather difficult to deal with. Therefore, an analytical solution at least for the part of such geometry is of engineering interest. Moreover, a trade-off between analytical and numerical approach clearly stressing out strength and weaknesses of both approaches is necessary.

The key parameter of the wire model is the current distribution induced along the electrode being excited by the equivalent current generator. The wire antenna model of the electrode is based on the homogeneous Pocklington integrodifferential equation. The scattered voltage along the electrode is obtained by integrating the scattered electric field generated by the induced current. The impact of a ground-air interface is taken into account via the corresponding Fresnel reflection coefficient (within the numerical solution approach) and simplified reflection coefficient approach arising from the modified image theory (within the analytical solution approach) [4]. The integro-differential relationships arising from the proposed wire antenna model are numerically treated by means of the GB-IBEM and analytically.

The scattered voltage along the electrode is obtained by integrating the current derivative along the electrode $[5,6]$.

\section{Formulation}

Vertical grounding electrode of length $L$ and radius $a$, buried in a lossy medium at depth $d$ and excited by an equivalent current source is shown in Fig 1.

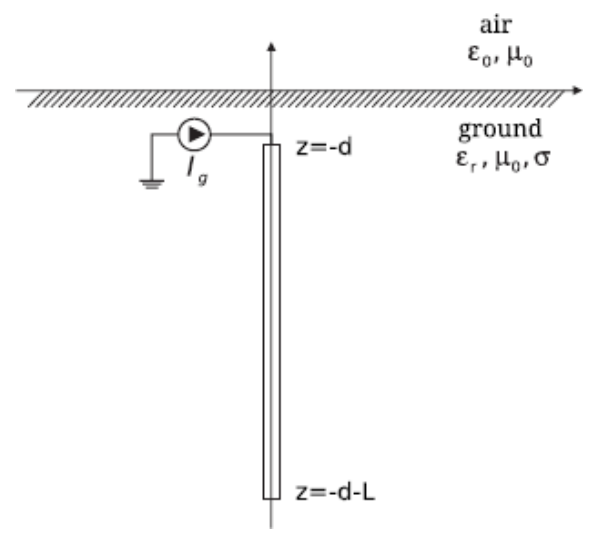

Figure 1: Vertical grounding electrode excited by the current source. 
The governing equations for the current and scattered voltage induced along the vertical electrode can be readily derived from Maxwell's equations by enforcing the continuity conditions for the tangential components of the electric field along the wire in the very similar manner as it was undertaken for the case of vertical electrode in [5] and horizontal electrode in [6].

A rigorous approach to account for the influence of lossy medium to the frequency response of the grounding electrode is related to the solution of Sommerfeld integrals, which is considered to be rather demanding and computationally expensive task [4-9].

\subsection{Integral equation for the current along the electrode}

The related homogeneous integro-differential equation of the Pocklington type for the current distribution induced along the electrode is given by

$$
\begin{aligned}
& j \omega \frac{\mu}{4 \pi} \int_{-d-L}^{-d} I\left(z^{\prime}\right) g\left(z, z^{\prime}\right) d z^{\prime} \\
& -\frac{1}{j 4 \pi \omega \varepsilon_{e f f}} \frac{\partial}{\partial z} \int_{-d-L}^{-d} \frac{\partial I\left(z^{\prime}\right)}{\partial z^{\prime}} g\left(z, z^{\prime}\right) d z^{\prime}+Z_{s}(z) I(z)=0
\end{aligned}
$$

where the complex permittivity of the lossy ground $\varepsilon_{e f f}$ is given by

$$
\varepsilon_{e f f}=\varepsilon_{r} \varepsilon_{0}+\frac{\sigma}{j \omega}
$$

with $\varepsilon_{r}$ and $\sigma$ being the soil permittivity and conductivity, respectively.

Furthermore, the Green function is of the form

$$
g\left(z, z^{\prime}\right)=g_{0}\left(z, z^{\prime}\right)-\Gamma_{r e f} g_{i}\left(z, z^{\prime}\right)
$$

while $g_{0}\left(z, z^{\prime}\right)$ denotes the lossy medium Green function

$$
g_{0}\left(z, z^{\prime}\right)=\frac{e^{-\gamma R_{1}}}{R_{1}}
$$

and $g_{i}\left(z, z^{\prime}\right)$ is, according to the image theory, given by

$$
g_{i}\left(z, z^{\prime}\right)=\frac{e^{-\gamma R_{2}}}{R_{2}}
$$

The propagation constant of the lossy ground is

$$
\gamma=\sqrt{j \omega \mu \sigma-\omega^{2} \mu \varepsilon_{r} \varepsilon_{0}}
$$

and $R_{1}, R_{2}$ are the distances from the source and the image to the observation point, respectively. 
The alternative approach, on the other hand, is related to the approximate reflection coefficient approach $[7,8]$, which is used in this paper as well.

Within the numerical solution the Fresnel reflection coefficient (RC) is used [2]

$$
\Gamma_{r e f}^{M I T}=\frac{\frac{1}{n} \cos \theta-\sqrt{\frac{1}{n}-\sin ^{2} \theta}}{\frac{1}{\underline{n}} \cos \theta+\sqrt{\frac{1}{\underline{n}}-\sin ^{2} \theta}} ; \theta=\operatorname{arctg} \frac{\left|x-x^{\prime}\right|}{2 d} ; \underline{n}=\frac{\varepsilon_{e f f}}{\varepsilon_{0}}
$$

Furthermore, a simplified reflection coefficient arising from modified image theory is used within the analytical solution [2-4]

$$
\Gamma_{r e f}^{M I T}=-\frac{\varepsilon_{e f f}-\varepsilon_{0}}{\varepsilon_{e f f}+\varepsilon_{0}}
$$

The electrode is excited via an equivalent ideal current source with one terminal connected to the electrode and the other one grounded at infinity, as presented in Fig 1. This current source is incorporated into the Pocklington equation formulation via the following conditions at the electrode ends [5]

$$
I(-d)=I_{g}, I(-d-L)=0
$$

where $I_{g}$ denotes the impressed unit current source.

It is worth emphasizing that the knowledge of the electrode current provides the assessment of the scattered voltage.

\subsection{The scattered voltage along the electrode}

The scattered voltage along the vertical electrode is defined by a line integral of the horizontal component of the scattered field (normal to the electrode and tangential to the ground-air interface) from the remote soil to the electrode surface [5]

$$
V^{s c t}(z)=-\int_{\infty}^{a} E_{x}^{s c t}(x, z) d x
$$

As the horizontal field component can be expressed in terms of the scalar potential gradient

$$
E_{x}^{s c t}=-\frac{\partial \phi}{\partial x}
$$

the scattered voltage along the electrode can be written as follows

$$
V^{s c t}(z)=\int_{\infty}^{a} \frac{\partial \varphi(x, z)}{\partial x} d x=\frac{d}{d z} \int_{\infty}^{a} \varphi(x, z) d x
$$


where $\varphi(x, z)$ is defined by the following particular integral

$$
\varphi(z)=\frac{1}{4 \pi \varepsilon_{e f f}} \int_{-d-L}^{-d} q\left(z^{\prime}\right) g\left(z, z^{\prime}\right) d z^{\prime}
$$

and the charge density is linked to current distribution through the continuity equation [4]

$$
q=-\frac{1}{j \omega} \frac{d I}{d z}
$$

Substituting continuity equation (14) into (13) yields

$$
\varphi(z)=-\frac{1}{j 4 \pi \omega \varepsilon_{e f f}} \int_{-d-L}^{-d} \frac{\partial I\left(z^{\prime}\right)}{\partial z^{\prime}} g\left(z, z^{\prime}\right) d z^{\prime}
$$

Inserting (15) into (12) and assuming the scalar potential in the remote soil to be zero [10], gives the scattered voltage along the electrode

$$
V^{s c t}(z)=-\frac{1}{j 4 \pi \omega \varepsilon_{e f f}} \int_{-d-L}^{-d} \frac{\partial I\left(z^{\prime}\right)}{\partial z^{\prime}} g\left(z, z^{\prime}\right) d z^{\prime}
$$

Note that the tedious integration from infinity to the electrode surface is avoided by taking the advantage of the very definition of voltage along the electrode (10).

\section{Solution}

The Pocklington equation (1) is solved numerically and analytically, respectively. The numerical solution is undertaken via the GB-IBEM [4].

\subsection{Numerical solution}

The unknown current $I^{e}\left(z^{\prime}\right)$ along the straight wire segment can be written as follows

$$
I^{e}\left(z^{\prime}\right)=\{f\}^{T}\{I\}
$$

Assembling the contributions from each segment, the Pocklington equation (1) is transferred into the matrix equation

$$
\sum_{j=1}^{M}[Z]_{j i}\{I\}_{i}=0, \text { and } j=1,2, \ldots, M
$$

where $M$ is the total number of segments and $[Z]_{j i}$ given by 


$$
\begin{aligned}
& {[Z]_{j i}=-\int_{\Delta l_{j}}\{D\}_{j} \int_{\Delta l_{i}}\left\{D^{\prime}\right\}_{i}^{T} g\left(z, z^{\prime}\right) d z^{\prime}+\int_{\Delta l_{j}}\{D\}_{j} \int_{\Delta l_{i}}\left\{D^{\prime}\right\}_{i}^{T} \Gamma_{r e f}^{F r} g_{i}\left(z, z^{\prime}\right) d z^{\prime}} \\
& +\gamma^{2} \int_{\Delta l_{j}}\{f\}_{j} \int_{\Delta l_{i}}\{f\}_{i}^{T} g\left(z, z^{\prime}\right) d z^{\prime} d z
\end{aligned}
$$

is the mutual impedance matrix representing the interaction of the $i$-th source with the $j$-th observation segment, respectively. The matrices $\{f\}$ and $\left\{f^{\prime}\right\}$ contain the shape functions while $\{D\}$ and $\left\{D^{\prime}\right\}$ contain their derivatives, and $\Delta l_{i}, \Delta l_{j}$ are the widths of $i$-th and $j$-th boundary elements.

A linear approximation over a wire segment is used in this work, as it was shown to be optimal in various EMC problems including thin wire configurations.

More details on the method can be found elsewhere, e.g. in [4].

\subsection{Analytical solution}

The Pocklington equation (1) can be solved analytically under certain conditions. It is convenient to write (1) in a following way

$$
-\frac{1}{j 4 \pi \omega \varepsilon_{e f f}}\left[\frac{\partial^{2}}{\partial z^{2}}-\gamma^{2}\right] \int_{-d-L}^{-d} \frac{\partial I\left(z^{\prime}\right)}{\partial z^{\prime}} g\left(z, z^{\prime}\right) d z^{\prime}+Z_{s}(z) I(z)=0
$$

The first step in the analytical solution of (20) is to write the integral on the left-hand side of (1) as follows

$$
\int_{-d-L}^{-d} I\left(z^{\prime}\right) g\left(z, z^{\prime}\right) d z^{\prime}=I(z) \int_{-d-L}^{-d} g\left(z, z^{\prime}\right) d z^{\prime}+\int_{-d-L}^{-d}\left[I\left(z^{\prime}\right)-I(z)\right] g\left(z, z^{\prime}\right) d z^{\prime}
$$

The integral on the left hand side of (21) is now approximated by the first term on the right hand side of (21), i.e. the second integral on the right hand side of (21) is neglected.

Furthermore, the solution of characteristic integral from (21) is

$$
\psi_{g}=\int_{h-\frac{L}{2}}^{h+\frac{L}{2}} g\left(z, z^{\prime}\right) d z^{\prime}=\int_{h-\frac{L}{2}}^{h+\frac{L}{2}} \frac{e^{-j k R}}{R} d z^{\prime}+\Gamma_{r e f}^{M I T} \int_{h-\frac{L}{2}}^{h+\frac{L}{2}} \frac{e^{-j k R_{i}}}{R_{i}} d z^{\prime}=2\left(\ln \frac{L}{a}-\Gamma_{r e f}^{M I T} \ln \frac{L}{2 d}\right)
$$

Consequently, the Pocklington equation (20) simplifies into the partial differential equation of the form

$$
\left[\frac{\partial^{2}}{\partial z^{2}}-\gamma_{g}^{2}\right] I(z)=0
$$

The analytical solution of (23) is

$$
I(z)=I_{g} \frac{\operatorname{sh}\left[\gamma_{e q}(d+L-z)\right]}{\operatorname{sh}\left(\gamma_{e q} L\right)}
$$


where

$$
\gamma_{e q}^{2}=\gamma^{2}+j 4 \pi \omega \frac{\varepsilon_{e f f}}{\psi_{g}} Z_{s}
$$

Furthermore, the scattered voltage along the electrode (16) is given by

$$
\begin{aligned}
& V^{s c t}(z)=-\frac{1}{j 4 \pi \omega \varepsilon_{e f f}} \int_{-d-L}^{-d} \frac{\partial I\left(z^{\prime}\right)}{\partial z^{\prime}} g\left(z, z^{\prime}\right) d z^{\prime} \\
& =\frac{\gamma_{e q} I_{g}}{j 4 \pi \omega \varepsilon_{e f f} \operatorname{sh}\left(\gamma_{e q} L\right)} \int_{-d-L}^{-d} c h \gamma_{e q}(d+L-z) g\left(z, z^{\prime}\right) d z^{\prime}
\end{aligned}
$$

and can be evaluated by means of numerical integration procedures.

\section{Results}

The first set of the results is related to the absolute value of spatial distribution of induced current computed numerically and analytically, while the second set deals with the results for the scattered voltage induced along the electrode and computed via both approaches, as well. The results are obtained for the following parameters of the electrode: $L=10 \mathrm{~m}, d=0.3 \mathrm{~m}, a=5 \mathrm{~mm}$. The excitation is the ideal current source $I_{g}=1 \mathrm{~A}$. Ground permittivity is $\varepsilon_{r}=10$. All Figures show the absolute value of the current distribution along the electrode.

Figure 2 shows the current distribution along the electrode at the operating frequency $f=10 \mathrm{MHz}$ for the ground conductivity $\sigma=0.01 \mathrm{~S} / \mathrm{m}$. The results computed analytically and via GB-IBEM agree rather satisfactorily.

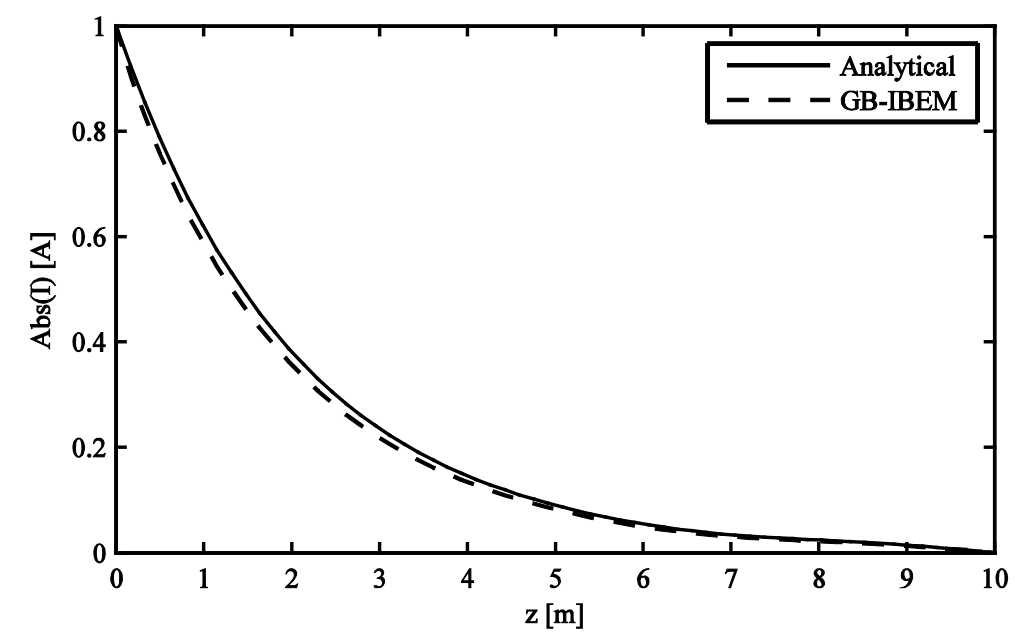

Figure 2: Current distribution (absolute value) along the vertical electrode for $f=10 \mathrm{MHz}$ and $\sigma=0.01 \mathrm{~S} / \mathrm{m}$. 
Figure 3 shows the current distribution along the same electrode at the frequency $f=10 \mathrm{MHz}$ for the ground conductivity $\sigma=0.001 \mathrm{~S} / \mathrm{m}$. The waveforms obtained via different approaches are still alike. However for the lower value of conductivity $(\sigma=0.001 \mathrm{~S} / \mathrm{m})$ differences in the results for the current distribution are slightly higher.

Figures 4 and 5 show the distribution of the scattered voltage induced along the same electrode for different values of ground conductivity at $f=10 \mathrm{MHz}$.

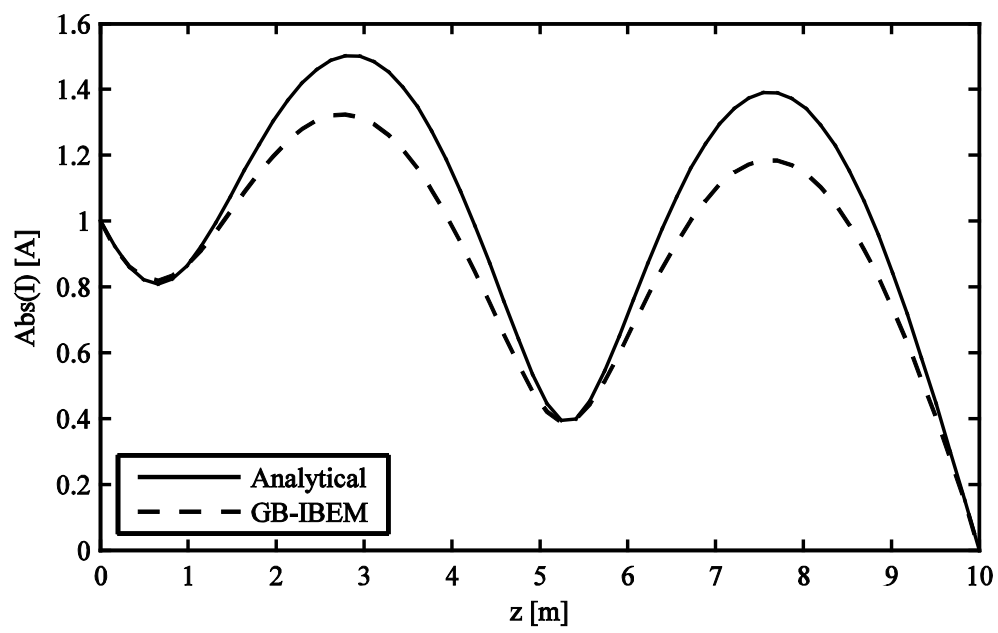

Figure 3: Current distribution (absolute value) along the vertical electrode for $f=10 \mathrm{MHz}$ and $\sigma=0.001 \mathrm{~S} / \mathrm{m}$.

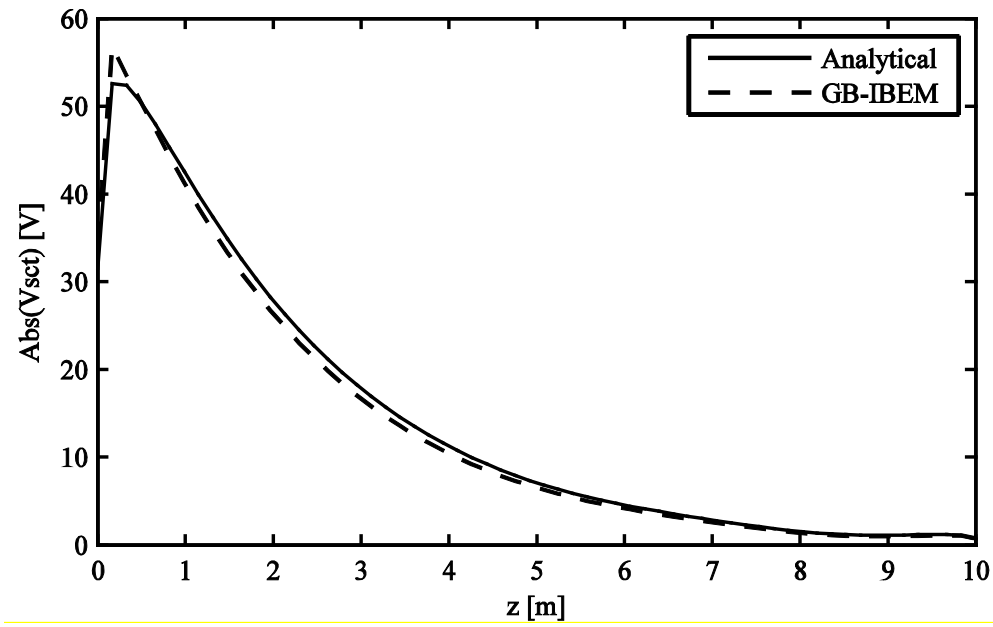

Figure 4: Scattered voltage distribution (absolute value) along the vertical electrode for $f=10 \mathrm{MHz}$ and $\sigma=0.01 \mathrm{~S} / \mathrm{m}$. 


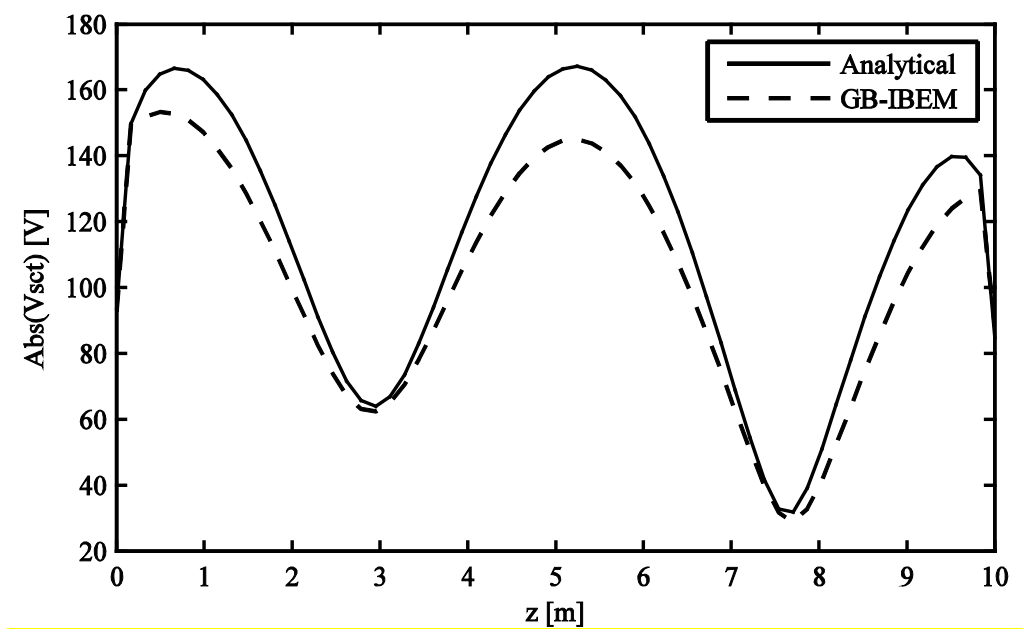

Figure 5: Scattered voltage distribution (absolute value) along the vertical electrode for $f=10 \mathrm{MHz}$ and $\sigma=0.001 \mathrm{~S} / \mathrm{m}$.

A satisfactory agreement between analytical and numerical results is achieved. Again, certain differences are noticeable at $f=10 \mathrm{MHz}$ for lower value of ground conductivity.

It is worth noting that the problem of the vertical electrode transient response in principle does not have a solution in the closed form. Consequently, the numerical solution is considered to be exact one, or at least more realistic in this case contrary to the usual cases (if analytical solution is available) where the goal of comparing numerical with analytical solutions is to show convergence of the numerical towards analytical results.

\section{Conclusion}

The paper deals with an analysis of vertical grounding electrode by means of straight wire antenna model featuring both numerical and analytical approach, respectively. The formulation is based on the solution of the homogeneous Pocklington integro-differential equation for half-space problems in the frequency domain. The presence of a lossy ground is taken into account via corresponding reflection coefficients. The numerical solution is carried out via the Galerkin-Bubnov scheme of the Indirect Boundary Element Method (GBIBEM). The same problem is also treated via the approximate analytical solution. Some illustrative computational examples are given in the paper. 


\section{References}

[1] D. Cavka, D. Poljak, V. Doric, R. Goic, Transient Analysis of Wind Turbines, Renewable Energy 43, pp. 284-291, 2012.

[2] D. Poljak, S. Sesnic, and R. Goic, "Analytical versus boundary element modelling of horizontal ground electrode," Engineering Analysis with Boundary Elements, 34, pp. 307-314, 2010.

[3] D. Cavka, D. Poljak, R. Goic, Transient Analysis of Ring Shaped Grounding Electrode using Isoparametric Quadratic Elements, Proc. ICLP 2010.

[4] D. Poljak, Advanced Modeling in Computational Electromagnetic Compatibility. New Jersey, USA: Wiley-Interscience, 2007.

[5] D. Poljak, V. Doric, "Wire antenna Model for Transient Analysis of Simple Grounding systems, Part I: The Vertical Grounding Electrode", Progress in Electromagnetics Research, PIER 64, pp. 149-166, 2006.

[6] D. Poljak, V. Doric, "Wire antenna Model for Transient Analysis of Simple Grounding systems, Part II: The Horizontal Grounding Electrode", Progress in Electromagnetics Research, PIER 64, pp. 149-166, 2006.

[7] E. K. Miller, A. J. Poggio, G. J. Burke, and E. S. Selden, "Analysis of wire Antennas in the Presence of a Conducting Half-Space. Part I. The Vertical Antenna in Free Space," Canadian Journal of Physics, 50, pp. 332-341, 1972.

[8] E. K. Miller, A. J. Poggio, G. J. Burke, and E. S. Selden, "Analysis of wire Antennas in the Presence of a Conducting Half-Space. Part II. The Horizontal Antenna in Free Space," Canadian Journal of Physics, 50, pp. 2614-2627, 1972.

[9] R. G. Olsen, M. C. Willis, "A Comparison of Exact and Quasi-static Methods for Evaluating Grounding Systems at High Frequencies", IEEE Trans. Power Delivery, 11(2), pp. 1071-1081, April 1996.

[10] F. M. Tesche, M. Ianoz, and T. Karlsson, EMC Analysis Methods and Computational Models. New York, USA: John Wiley \& Sons, Inc., 1997. 